\title{
Do food bioactives play a role in attention-deficit/ hyperactivity disorder?
}

\author{
Klaus W. Lange*
}

Department of Experimental Psychology, University of Regensburg, Germany

${ }^{*}$ Corresponding author: Klaus W. Lange, Institute of Psychology, University of Regensburg, 93040 Regensburg, Germany. Tel: +49 941 9433815; Fax: +49 941 9434496; E-mail: klaus.lange@ur.de

DOI: $10.31665 / J F B .2018 .4160$

Received: December 9, 2018; Revised received \& accepted: December 17, 2018

Citation: Lange, K.W. (2018). Do food bioactives play a role in attention-deficit/hyperactivity disorder?. J. Food Bioact. 4: 1-7.

\begin{abstract}
Attention-deficit/hyperactivity disorder (ADHD) is a common diagnosis in children, which may persist into adulthood. Short-term benefits of pharmacological treatment have been shown, but concerns regarding long-term efficacy and safety have led to a search for alternative treatments. Nutrition may be an important factor in psychiatric disorders. At group level, food bioactives do not appear to play a significant pathogenetic or therapeutic role in ADHD. Benefits of treatment with omega-3 fatty acids, vitamins and minerals may be confined to individuals deficient in these micronutrients. Dietary patterns may be of greater importance than individual nutrients. However, both diet and consumption of certain nutrients may be precursors or consequences of ADHD. The controversy surrounding the clinical and pathogenetic heterogeneity of ADHD complicates the assessment of food bioactives. The identification of a potential role of food bioactives in ADHD is hindered by the ill-defined nature of the disorder and the lack of biological markers underpinning its validity. The therapeutic efficacy of food bioactives cannot be tested by evaluating their effect on the pathophysiology of ADHD but only by observing their effects on symptom severity.
\end{abstract}

Keywords: Attention-deficit/hyperactivity disorder; Diet; Food bioactives; Omega-3 fatty acids; Minerals; Vitamins.

\section{Introduction}

ADHD is a diagnostic category described in the Diagnostic and Statistical Manual of Mental Disorders (DSM) of the American Psychiatric Association (American Psychiatric Association, 1994, 2013a) and is one of the most common psychiatric diagnoses in childhood and adolescence. Substantial comorbidity of ADHD with other psychiatric disorders exists. The prevalence of ADHD has been estimated to be $5.29 \%$ of the world's childhood population (Polanczyk et al., 2007). Significant variability in rates of ADHD in various studies has been claimed to be due to methodological problems rather than differences in the actual distribution of ADHD (Polanczyk et al., 2007). It is widely assumed that ADHD is a neurodevelopmental disorder, which begins in childhood and can persist into adulthood. The findings of longitudinal studies suggest that two-thirds of youths diagnosed with ADHD will continue to present with symptoms of ADHD in adulthood.

Medication and behavior therapy have been demonstrated to yield short-term symptom reduction in children, adolescents, and adults with ADHD. Psychostimulants can improve attention and reduce activity levels in children in the short term, but they have little impact on academic performance or quality of life (KortekaasRijlaarsdam et al., 2018; Loe and Feldman, 2007), and initial symptomatic effects are not sustained on long-term follow-up (Jensen et al., 2007; Swanson et al., 2017). Tolerance to stimulants may decrease treatment efficacy in the course of long-term use (Wang et al., 2013). The uncritical expansion of the use of ADHD medications into the treatment of adults based on the findings in children has been criticized (Bijl, 2014, 2018). The maturing brain depends on critical developmental processes and is therefore sensitive to pharmacological interventions (Swaab and Boer, 2001). A careful analysis of the safety of ADHD medication in children and adolescents is therefore needed. The administration of common ADHD medica- 
Table 1. Problems surrounding a role of food bioactives in ADHD

\section{General problems of ADHD research}

No biological or neuropsychological markers; no evidence of brain disorder.

Diagnosis based on subjective assessment of symptoms.

Validity and reliability of descriptive approach to diagnosis?

Overdiagnosis of ADHD due to nonspecific attention problems.

High rates of comorbid disorders.

Heterogeneous and non-representative study samples.

Summary: ADHD is an ill-defined and clinically heterogeneous condition.

\section{Findings on medication in ADHD}

Short-term improvements in ratings of core symptoms.

Assessment of effects only on symptoms, not on pathophysiology.

Concerns regarding effects on clinically and ecologically relevant outcome measures.

Concerns regarding long-term efficacy (>12 weeks).

Decreased efficacy of long-term use due to tolerance?

Concerns regarding long-term safety; adverse events in adults?

Summary: Inconclusive evidence of benefits and harms of medication; alternative treatment options needed.

\section{Findings on food bioactives in ADHD}

Omega-3 fatty acids, vitamins, and minerals are main bioactives analyzed.

Correlations between food bioactives and presence or severity of symptoms (causality?).

Several limitations of available studies.

No long-term randomized controlled treatment trials available.

No evidence of a role of single bioactives in pathogenesis or treatment of ADHD.

Benefits of certain food bioactives confined to people with micronutrient deficiencies?

Optimal dosages, combinations, and durations of administration?

Critical phases of brain development for bioactives?

Use of outcome measures including functioning, quality of life, and adaptive skills needed.

Large-scale, prospective, randomized controlled trials of food bioactives needed.

Dietary patterns may be of greater importance than individual nutrients.

Summary: Inconclusive evidence of role of individual food bioactives; dietary patterns and interaction with other lifestyle factors may be more important.

tions may be associated with various adverse effects as serious as growth retardation and severe cardiovascular events (Lange, 2017a, 2018a; Storebø et al., 2018; Swanson et al., 2017). The present state of information based on randomized controlled trials may indicate that pharmacotherapy for ADHD should be administered with caution or even discontinued after a period of 12 weeks (Lange, 2018a) and alternative treatment strategies should be explored.

The major points discussed in the present viewpoint are summarized in Table 1.

\section{Dietary patterns and food bioactives in ADHD}

Nutritional and dietary approaches to the treatment of mental dis- orders have attracted considerable scientific interest in recent years (Sarris et al., 2015), and several food bioactives, with roles in the support of brain function, have been postulated to play an important role in the etiology and treatment of mental disorders (Marx et al., 2017). A sufficient supply of nutrients during brain development is critical. Dysfunctioning of the prefrontal cortex, which continues to develop until adolescence, is believed to be involved in the pathogenesis of ADHD, and diets deficient in essential nutrients may contribute to the occurrence of ADHD symptoms (Glanzman, 2009). Food bioactives that have been linked to the etiology or pathophysiology of ADHD and claimed to be of potential therapeutic use are vitamins, trace elements, and polyunsaturated fatty acids (PUFAs).

Several studies have examined the role of whole diets and di- 
etary patterns in ADHD. For example, in a large population-based cohort study, it was found that a higher score for a 'Western' dietary pattern was associated with ADHD diagnosis after adjusting for known confounding factors, while ADHD diagnosis was not associated with a 'healthy' dietary pattern (Howard et al., 2011). A case-control study found that a fish/white meat dietary pattern was inversely related to ADHD in children and adolescents with ADHD (Zhou et al., 2016). An inverse association was also observed for a nutrient pattern rich in zinc, protein and other minerals (Zhou et al., 2016). An increase in the prevalence of ADHD diagnosis was associated with low adherence to a Mediterranean diet (Rios-Hernandez et al., 2017). A positive correlation between hyperactivity and the consumption of processed meat and salty snacks was reported, while a negative correlation was found between hyperactivity and the intake of vegetables, coarse cereals, aquatic foods, beef, mutton, and milk (Liu et al., 2014). The observational designs used in the above studies allow no conclusions regarding causal relationships between dietary patterns and ADHD. For example, preference for certain diets could be a consequence of ADHD behaviors. In addition, many interactions and overlaps exist between diet, nutrition and other lifestyle factors, such as physical activity (Lange 2018b, c). For example, significant cognitive benefits in people on a Mediterranean diet (Valls-Pedret et al., 2015) were found in highly active people living in a Mediterranean culture. Such findings limit the external generalizability of the findings on diet.

Omega-3 fatty acids are structural and functional components of cell membranes, which play an important role in brain functioning. In view of dietary patterns in Western countries, with high amounts of processed foods containing low levels of omega-3 fatty acids, a discrepancy between desirable and actual consumption of bioactives in both children and adults could be expected (see Lange, 2017b; Lange et al., 2014a, 2017). Trials assessing the therapeutic efficacy of omega-3 PUFAs were conducted in ADHD sufferers with decreased blood levels of these compounds (Lange et al., 2014a). A systematic review of metaanalyses of double-blind placebo-controlled trials showed that the effect sizes for PUFA supplementation were small in regard to the ratings of ADHD symptoms by parents and teachers (Pelsser et al., 2017). A recent randomized placebo-controlled trial evaluating the efficacy of omega-3 PUFAs in children and adolescents with ADHD over three months found that the total reduction in an ADHD rating score was significantly greater in the placebo group than in the PUFA group (Cornu et al., 2018). Moreover, the pooling of these data with previously published results demonstrated no overall effect of omega-3 PUFAs on ADHD symptoms (Cornu et al., 2018).

Several mechanisms explaining a relationship between ADHD and micronutrients have been proposed. Various vitamins and minerals serve as important cofactors of enzymes required in the metabolic steps involved in the synthesis, uptake, and breakdown of neurotransmitters in the brain. Metabolic dysfunction associated with a reduction in the availability of vitamin and mineral cofactors is a possible mechanism underlying psychiatric symptoms (Ames et al., 2002). Multinutrient supplementation may provide sufficient cofactors to saturate even enzymes with significantly reduced activity and thus restore undisturbed function (Ames et al., 2002). Improved energy metabolism has also been hypothesized as an explanation for the effects of nutrients on the brain (Arnold et al., 2007). It is unclear whether vitamin (e.g. B vitamins) or mineral (e.g. zinc, magnesium, iron) deficiencies are, in fact, involved in the pathophysiology of ADHD, and the evidence regarding therapeutic efficacy of their supplementation is inconclusive (e.g. Hariri and Azadbakht, 2015; Lange et al., 2017).

\section{Limitations of studies investigating food bioactives in ADHD}

Observational studies demonstrating correlations between food bioactive levels (e.g. in blood serum) in individuals with ADHD and the presence or severity of ADHD symptoms may help generate hypotheses regarding associations but do not allow any conclusions on causality. Several studies on food bioactives in ADHD have used study designs lacking an appropriate control group. Thus, any effects observed may simply be a result of the placebo effect. Randomized controlled trials are the gold standard in the hierarchy of evidence regarding the efficacy of therapeutic interventions (Devereaux and Yusuf, 2003). Such long-term randomized controlled trials assessing food bioactives in ADHD are largely absent.

Other major limitations of the available studies on food bioactives in ADHD are relatively small sample sizes, varied sampling procedures and inclusion criteria as well as multiple assessment methods. Studies assessing the effects of bioactives have used mainly samples of male children, and generalization of their findings to adults and females requires caution. In addition, many trials have excluded individuals with comorbidities, frequently rendering the trial samples different from populations of people with ADHD in the community.

Future studies could address the above issues and large-scale, sufficiently powered, blinded, prospective, randomized controlled trials evaluating the impact of food bioactives should be conducted. However, a range of specific problems related to the investigation of the etiological and therapeutic involvement of food bioactives in ADHD and other psychiatric disorders must be considered.

\section{Problems of investigating food bioactives in ADHD}

Researchers interested in food bioactives and their effects on mental health are seldom acquainted with the difficulties surrounding the classification, diagnosis, and therapy of mental disorders such as ADHD. Food scientists usually accept the validity of the concept of ADHD and are unaware of the manifold problems in the investigation and interpretation of the effects of food bioactives in ADHD.

Although the description of ADHD in international classification systems (American Psychiatric Association, 1994, 2013a) seems to reflect a consensus regarding the clinical entity of ADHD, considerable controversy surrounding this issue remains (Lange et al., 2010). ADHD is a clinically heterogeneous condition. Children presenting with what is considered to be poor concentration (sustained attention), overactivity, and impulsivity are typically diagnosed with ADHD. The diagnosis is based on the grouping of symptom presentation, which can change over time. For example, the subtypes of ADHD present in DSM-IV (American Psychiatric Association, 1994) were abandoned in DSM-5 (American Psychiatric Association, 2013a), having been shown, with the passage of time, to lack diagnostic stability. The diagnostic procedures used in ADHD are based largely, if not exclusively, on subjective assessments of perceived behavior. The descriptive approach to diagnosis, as used by the DSM to characterize ADHD based on self-reports or informant-reports, may not always be valid or reliable. The assumption that ADHD is a valid and useful diagnosis in children and adolescents has been disputed on many grounds, including lack of biological or psychological markers, high rates of comorbid disorders, inconsistent clustering of symptoms, and differing cultural perceptions (Timimi and Leo, 2009). Problems of attention and restlessness are non-specific behaviors and are 
commonly found in other psychiatric disorders and in the "normal' population. ADHD may be viewed as falling on a continuum of behavior rather than as a discrete 'brain malfunction'. Serious adverse outcomes commonly associated with ADHD may be more strongly related to co-occurring problems such as conduct disorder (Barkley et al., 2004). Significant increases in the rate of ADHD diagnoses have been reported (e.g. Davidovitch et al. 2017). This has been attributed to changing attitudes towards diagnosing ADHD among physicians and parents, with parents increasingly considering the diagnosis and treatment of ADHD as a means to improve their children's academic performance. It has been argued that ADHD is over-diagnosed and over-treated with medication in high-income and middle-income countries (Frances and Batstra, 2013; Halldner et al., 2014). The assumption that ADHD is a disorder beginning in childhood and persisting into adulthood has recently been questioned (Apter, 2018), since $90 \%$ of adult cases of ADHD were found to lack a history of childhood ADHD (Moffitt et al., 2015). In addition, doubt has been cast on the very concept of ADHD in adults. While some authors have argued in favor of its validity (Asherson et al., 2010), others have emphasized its vagueness and the lack of evidence for the value of specific treatments (Moncrieff and Timimi, 2010). The claims that ADHD is a life-long disorder requiring life-long therapy (Kooij et al., 2010) are supported largely by experts sponsored by the pharmaceutical industry and are promoted through aggressive marketing (Moncrieff and Timimi, 2010). Exponents of the concept of ADHD have made great efforts to downplay the possibility that social, cultural, and environmental factors play any role in the diagnosis or prevalence of the disorder (see Smith, 2017). The current bias towards biological approaches to ADHD, at the cost of lifestyle and social interventions, is heavily promoted by the profit-dependent pharmaceutical industry, since biological explanations of ADHD may create pressure to accept pharmacological solutions.

The characterising of behavior on the spectrum of normal biological variation as indicative of specific somatic pathology and the consequent labelling of a portion of the population as "disturbed" is unwarranted. It is necessary to establish a demonstrable difference between individuals diagnosed with ADHD and 'healthy' people. No distinctive etiology, pathophysiology, biomarker or cognitive profile has been identified (Sontag et al., 2010; Thome et al., 2012; Lange et al., 2014b). Breakthroughs in the genetics of ADHD have been promised for many years and an impressive number of genes have been posited to be involved in ADHD (Faraone and Larsson, 2018). However, the small odds ratios for the genes associated with ADHD suggest that many genes could be involved and that these genes may each have a small effect (Faraone and Larsson, 2018). The American Psychiatric Association has officially admitted that no genes have, thus far, been found for the disorders described in its manual (American Psychiatric Association, 2013b). It was further conceded that the decades-old promise of an identification of mental disorders based on genetic and biological markers providing precise, valid, and reliable diagnoses has not been fulfilled (American Psychiatric Association, 2013b). Claims presuming faulty genes and neurochemical imbalances in the brains of individuals with psychiatric disorders including ADHD are, thus, not supported by actual scientific findings. While the 'missing heritability' position assumes that the failure to identify genes for mental disorders is the result of a lack of suitable research methods, an alternative explanation for this failure is that the genes claimed to underlie mental disorders do not exist (Joseph, 2012).

While neuroimaging techniques are vital in the diagnosis of brain tumors, they are not useful tools in the diagnosis or therapeutic evaluation of ADHD. An ever-increasing number of investiga- tions using more sophisticated imaging techniques and postulating an ever-expanding number of potentially problematic brain regions in ADHD, with little reproducibility between studies, demonstrate the limited value of these techniques in the the diagnosis of ADHD (Cohen and Leo, 2004). For example, a widely publicized but seriously flawed and much criticized imaging study claimed to have found brain alterations in ADHD. The large-scale analysis reported smaller volumes of several subcortical brain regions as well as a decrease in intracranial volume in individuals with ADHD compared to healthy controls (Hoogman et al., 2017). The authors of this study concluded that individuals with ADHD have altered brains and presented their findings as proof that ADHD is a disorder of the brain (Hoogman et al., 2017). However, as has been pointed out (Batstra et al., 2017; Bejerot et al., 2017; Dehue et al., 2017; Poulton and Nanan, 2017), such a definitive conclusion is not supported by the data. Considerable overlap between groups and large within-group variation were found (Dehue et al, 2017). The effect sizes ranging from -0.19 to -0.10 for the differences in volume indicate that approximately $95 \%$ of the two groups overlap, which is normally interpreted as a negligible or very small difference (Batstra et al., 2017). In addition, the results showed no significant differences in the brains of adults with ADHD compared to those without the diagnosis (Dehue et al., 2017). Moreover, even assuming the existence of such associations, these do not necessarily imply causality, since environmental factors may change brain anatomy. Factors confounding the results include the intelligence quotient (IQ) (Bejerot et al, 2017). When IQ difference was controlled for, no significant differences between individuals with ADHD and controls could be found in any of the investigated brain regions (Bejerot et al., 2017). Furthermore, the involvement of brain areas associated with emotional regulation may suggest that the volume differences observed may be related to subclinical symptoms of oppositional defiant disorder, which is commonly associated with ADHD (Poulton and Nanan, 2017). In summary, the speculative claim that a reduced volume of certain brain regions may be seen in individuals with ADHD is belied by the available data. There is currently no proof of a brain disorder in children with ADHD. It has been shown that the youngest children in a classroom are the ones most likely to be diagnosed with ADHD, possibly because their brains are less developed, which hardly justifies the assumption of a brain disorder (Halldner et al., 2014). Alternative explanations of ADHD, which are often disparaged because they lend no support to the prevailing biological paradigm of ADHD (Visser and Jehan, 2009), should therefore be considered. The question of whether ADHD is a bone fide brain disorder or a social construction remains to be answered (see Dehue, 2014).

In summary, attempts to pinpoint biological deficits in individuals diagnosed with $\mathrm{ADHD}$, using a wide range of research strategies, such as genetics, brain imaging or neuropsychological analysis, have thus far been without success. The efficacy of treatments including food bioactives cannot, therefore, be tested in regard to their effect on the pathophysiology of ADHD. The therapeutic effects of food bioactives can be assessed only through an evaluation of their efficacy in reducing indicators of symptom severity.

Current treatments may reduce the negative impact of untreated ADHD on life functioning but do not usually lead to "normalization' of behavior (Shaw et al., 2012). The assessment of treatment outcomes in $\mathrm{ADHD}$, which is commonly confined to symptom rating, needs to incorporate measures of functioning, quality of life, and adaptive skills, especially when long-term treatment response is investigated (Epstein and Weiss, 2012). ADHD can have a substantial social impact on patients' lives and may affect education, employment, finances, and social relationships. This should be taken into account when defining outcome measures beyond the 
core symptoms of ADHD. Statistically significant improvements on symptom rating scales do not necessarily translate into clinically and ecologically relevant outcomes related to the problems of daily life.

It has been proposed that a sufficiency of nutrients during critical periods of brain development is essential and supplementation after this time may be of limited benefit. Nutritional deficits may have a particularly important effect on brain development between weeks 24 and 42 of gestation, during which myelination and synapse formation take place (Georgieff, 2007). The investigation of the effects of single nutrients at individual periods of time under controlled conditions may therefore not account for the need of the brain for a variety of substances in adequate amounts and proportions at critical times. Food bioactives needed during late fetal and early neonatal life include protein, zinc, iron, copper, choline, and PUFAs (Georgieff, 2007). Prenatal exposure to 'unhealthy diets' has been shown to be associated with ADHD symptoms (Rijlaarsdam et al., 2017). Furthermore, low maternal serum vitamin D levels during the first trimester have been associated with ADHD-like symptoms in preschool-age children (Daraki et al., 2017). Correlations between adherence of children to a healthy diet and low rates of ADHD do not necessarily point to protective effects of a healthy diet in childhood. The mothers of children consuming healthy foods may also have adhered to healthy diets during pregnancy, providing essential nutrients to the child at critical stages of brain development, thus potentially avoiding deficits that could later lead to behavioral problems.

\section{Conclusions}

Evidence supporting a role of single nutrients in the pathogenesis and treatment of ADHD has so far not been produced. Benefits of certain food bioctives may be confined to individuals with nutritional deficiencies. Since ADHD is a complex, heterogeneous disorder, multi-ingredient supplementation may be necessary to achieve beneficial effects. Unwanted side effects of food supplements should be considered. These may become apparent many years after administration and thus elude detection. The adherence to certain dietary patterns may provide better predictors of disease risk than the analysis of single nutrients or foods (Hu, 2002). Outcomes for healthy diets may be better than for individual nutrients due to the cumulative beneficial effects of their many ingredients. However, establishing adherence to medium and longer term dietary interventions beyond the administration of supplements may not be feasible. It should also be considered that dietary patterns observed in individuals diagnosed with ADHD could be a consequence rather than a cause of ADHD.

Current evidence suggests that nutrition plays a key role in brain development, mental health and psychiatric disorders including ADHD. However, the many difficulties in assessing the effects of specific food bioactives, such as identifying useful compounds, combining them at optimal dosages, determining the necessary durations of administration, and investigating the critical phases of brain development for bioactives, may appear insurmountable. A main obstacle in the investigation is the heterogeneous nature and problematic classification of ADHD. The development of an objective, reliable and biologically based classification of ADHD is urgently needed. Substantial progress in revealing the role of food bioactives in the pathogenesis and therapy of ADHD will remain elusive as long as psychiatrists fail to acknowledge that ADHD is an ill-defined construct and are unable to identify biological or neuropsychological markers underpinning its validity. The therapeutic efficacy of food bioactives in ADHD cannot therefore be evaluated according to their effects on objective pathophysiological measures but only by subjective rating of symptoms, the clinical relevance of which is questionable. Statistically significant improvements on symptom rating scales do not necessarily equate with clinically and ecologically relevant outcomes related to the problems of daily life faced by those with ADHD.

\section{References}

American Psychiatric Association. (1994). Diagnostic and statistical manual of mental disorders, 4th edition. American Psychiatric Association, Washington, American Psychiatric Association.

American Psychiatric Association. (2013a). Diagnostic and statistical manual of mental disorders, 5th edition. American Psychiatric Association, Washington, American Psychiatric Association.

American Psychiatric Association. (2013b). Chair of DSM-5 task force discusses future of mental health research. Statement by David Kupfer, M.D. American Psychiatric Association, American Psychiatric Association Press release No. 13-33 (May 3, 2013).

Ames, B.N., Elson-Schwab, I., and Silver, E.A. (2002). High-dose vitamin therapy stimulates variant enzymes with decreased coenzyme binding affinity (increased $\mathrm{Km}$ ): relevance to genetic disease and polymorphisms. Am. J. Clin. Nutr. 75: 616-658.

Apter, A. (2018). Are childhood and adult ADHD the same entities. Eur. Child Adolesc. Psychiatry 27: 821-822.

Arnold, L.E., Amato, A., Bozzolo, H., Hollway, J., Cook, A., Ramadan, Y., Crowl, L., Zhang, D., Thompson, S., Testa, G., Kliewer, V., Wigal, T., McBurnett, K., and Manos, M. (2007). Acetyl-L-carnitine in attentiondeficit/hyperactivity disorder: a multi-site, placebo-controlled pilot trial. J. Child Adolesc. Psychopharmacol. 17: 791-802.

Asherson, P., Adamou, M., Bolea, B., Müller, U., Dunn Morua, S., Pitts, M., Thome, J., and Young, S. (2010). Is ADHD a valid diagnosis in adults? Yes. BMJ 340: c549.

Barkley, R.A., Fischer, M., Smallish, L., and Fletcher, K. (2004). Young adult follow-up of hyperactive children: antisocial activities and drug use. J. Child Psychol. Psychiatry 45: 195-211.

Batstra, L., te Meerman, S., Conners, K., and Frances, A. (2017). Subcortical brain volume differences in participants with attention deficit hyperactivity disorder in children and adults. Lancet Psychiatry 4: 439.

Bejerot, S., Nilsonne, G., and Humble, M.B. (2017). Subcortical brain volume differences in participants with attention deficit hyperactivity disorder in children and adults. Lancet Psychiatry 4: 437.

Bijl, D. (2014). Promoting drugs through research papers: methylphenidate for ADHD in adults. Geneesmiddelenbull. 48(9): 105-106.

Bijl, D. (2018). Het pillenprobleem. Waarom we zoveel medicijnen gebruiken die niet werken en niet helpen. Amsterdam University Press, Amsterdam.

Cohen, D., and Leo, J. (2004). An update on ADHD neuroimaging research. J. Mind Behav. 25: 161-166.

Cornu, C., Mercier, C., Ginhoux, T., Masson, S., Mouchet, J., Nony, P., Kassai, B., Laudy, V., Berquin, P., Franc, N., Le Heuzey, M.F., Desombre, H., and Revol, O. (2018). A double-blind placebo-controlled randomised trial of omega-3 supplementation in children with moderate ADHD symptoms. Eur. Child Adolesc. Psychiatry 27: 377-384.

Daraki, V., Roumeliotaki, T., Koutra, K., Chalkiadaki, G., Katrinaki, M., Kyriklaki, A., Kampouri, M., Margetaki, K., Vafeiadi, M., Papavasiliou, S., Kogevinas, M., and Chatzi, L. (2018). High maternal vitamin D levels in early pregnancy may protect against behavioral difficulties at preschool age: the Rhea mother-child cohort, Crete, Greece. Eur. Child Adolesc. Psychiatry 27: 79-88.

Davidovitch, M., Koren, G., Fund, N., Shrem, M., and Porath, A. (2017). Challenges in defining the rates of ADHD diagnosis and treatment: trends over the last decade. BMC Pediatrics 17: 218.

Dehue, T. (2014). Betere mensen: over gezondheid als keuze en koopwaar. Atlas-Contact, Amsterdam.

Dehue, T., Bijl, B., de Winter, M., Scheepers, F., Vanheule, S., van Os, J., Verhaeghe, P., and Verhoeff, B. (2017). Subcortical brain volume differences in participants with attention deficit hyperactivity disorder 
in children and adults. Lancet Psychiatry. 4: 438-439.

Devereaux, P.J., and Yusuf, S. (2003). The evolution of the randomized controlled trial and its role in evidence-based decision making. J. Intern. Med. 254: 105-113.

Epstein, J.N., and Weiss, M.D. (2012). Assessing treatment outcomes in attention-deficit/hyperactivity disorder: a narrative review. Prim. Care Companion CNS Disord. 14: PCC.11r01336.

Faraone, S.V., and Larsson, H. (2018). Genetics of attention deficit hyperactivity disorder. Mol. Psychiatry doi:10.1038/s41380-018-0070-0.

Frances, A., and Batstra, L. (2013). Why so many epidemics of childhood mental disorder? J. Dev. Behav. Pediatr. 34: 291-292.

Georgieff, M.K. (2007). Nutrition and the developing brain: nutrient priorities and measurement. Am. J. Clin. Nutr. 85: 614S-620S.

Glanzman, M.M. (2009). ADHD and nutritional supplements. Curr. Atten. Disord. Rep. 1: 75-81.

Halldner, L., Tillander, A., Lundholm, C., Boman, M., Långström, N., Larsson, H., and Lichtenstein, P. (2014). Relative immaturity and ADHD: findings from nationwide registers, parent- and self-reports. J. Child Psychol. Psychiatry 55: 897-904.

Hariri, M., and Azadbakht, L. (2015). Magnesium, iron, and zinc supplementation for the treatment of attention deficit hyperactivity disorder: a systematic review on the recent literature. Int. J. Prev. Med. 6: 83.

Hoogman, M., and ENIGMA ADHD Working Group. (2017). Subcortical brain volume differences of participants with ADHD across the lifespan: an ENIGMA collaboration. Lancet Psychiatry 4: 310-319.

Howard, A.L., Robinson, M., Smith, G.J., Ambrosini, G.L., Piek, J.P., and Oddy, W.H. (2011). ADHD is associated with a "Western" dietary pattern in adolescents. J. Atten. Disord. 15: 403-411.

Hu, F.B. (2002). Dietary pattern analysis: a new direction in nutritional epidemiology. Curr. Opin. Lipidol. 13: 3-9.

Jensen, P.S., Arnold, L.E., Swanson, J.M., Vitiello, B., Abikoff, H.B., Greenhill, L.L., Hechtman, L., Hinshaw, S.P., Pelham, W.E., Wells, K.C., Conners, C.K., Elliott, G.R., Epstein, J.N., Hoza, B., March, J.S., Molina, B.S., Newcorn, J.H., Severe, J.B., Wigal, T., Gibbons, R.D., and Hur, K. (2007). 3-year follow-up of the NIMH MTA study. J. Am. Acad. Child Adolesc. Psychiatry 46: 989-1002.

Joseph, J. (2012). The "missing heritability" of psychiatric disorders: elusive genes or non-existent genes? Appl. Dev. Sci. 16: 65-83.

Kooij, S.J., Bejerot, S., Blackwell, A., Caci, H., Casas-Brugué, M., Carpentier, P.J., Edvinsson, D., Fayyad, J., Foeken, K., Fitzgerald, M., Gaillac, V., Ginsberg, Y., Henry, C., Krause, J., Lensing, M.B., Manor, I., Niederhofer, H., Nunes-Filipe, C., Ohlmeier, M.D., Oswald, P., Pallanti, S., Pehlivanidis, A., Ramos-Quiroga, J.A., Rastam, M., Ryffel-Rawak, D., Stes, S., and Asherson, P. (2010). European consensus statement on diagnosis and treatment of adult ADHD: The European Network Adult ADHD. BMC Psychiatry 10: 67.

Kortekaas-Rijlaarsdam, A.F., Luman, M., Sonuga-Barke, E., and Oosterlaan, J. (2018). Does methylphenidate improve academic performance? A systematic review and meta-analysis. Eur. Child Adolesc. Psychiatry. doi:10.1007/s00787-018-1106-3.

Lange, K.W. (2017a). The treatment of attention deficit hyperactivity disorder has no proven long-term benefits but possible adverse effects. Mov. Nutr. Health Dis. 1: 11-25.

Lange, K.W. (2017b). Dietary factors in the etiology and therapy of attention deficit/hyperactivity disorder. Curr. Opin. Clin. Nutr. Metab. Care 20: 464-469.

Lange, K.W. (2018a). The evidence of the benefits and harms of methylphenidate in the treatment of attention deficit/hyperactivity disorder is inconclusive. J. Pharmacol. Clin. Toxicol. 6: 1118.

Lange, K.W. (2018b). Lifestyle and attention deficit/hyperactivity disorder. Mov. Nutr. Health Dis. 2: 22-30.

Lange, K.W. (2018c). Diet, exercise, and mental disorders - public health challenges of the future. Mov. Nutr. Health Dis. 2: 39-59.

Lange, K.W., Reichl, S., Lange, K.M., Tucha, L., and Tucha, O. (2010). The history of attention deficit hyperactivity disorder. Atten. Defic. Hyperact. Disord. 2: 241-255.

Lange, K.W., Hauser, J., Kanaya, S., Kaunzinger, I., Lange, K.M., MakulskaGertruda, E., Nakamura, Y., Sontag, T.A., and Tucha, L. (2014a). Polyunsaturated fatty acids in the treatment of attention deficit hyperactivity disorder. Funct. Foods Health Dis. 4: 245-253.

Lange, K.W., Hauser, J., Lange, K.M., Makulska-Gertruda, E., Takano, T.,
Takeuchi, Y., Tucha, L., and Tucha, O. (2014b). Utility of cognitive neuropsychological assessment in attention-deficit/hyperactivity disorder. Atten. Defic. Hyperact. Disord. 6: 241-248.

Lange, K.W., Hauser, J., Lange, K.M., Makulska-Gertruda, E., Nakamura, Y., Reissmann, A., Sakaue, Y., Takano, T., and Takeuchi, Y. (2017). The role of nutritional supplements in the treatment of ADHD: what the evidence says. Curr. Psychiatry Rep. 19: 8.

Liu, J., He, P., Li, L., Shen, T., Wu, M., Hu, J., Zhuang, Y., Yin, J., and He, G. (2014). Study on the association between diet, nutrient and attention deficit hyperactivity disorder among children in Shanghai, Kunshan, Wuxi three kindergarten. Wei Sheng Yan Jiu 43: 235-239.

Loe, I.M., and Feldman, H.M. (2007). Academic and educational outcomes of children with ADHD. J. Pediatr. Psychol. 32: 643-654.

Marx, W., Moseley, G., Berk, M., and Jacka, F. (2017). Nutritional psychiatry: the present state of the evidence. Proc. Nutr. Soc. 76: 427-436.

Moffitt, T.E., Houts, R., Asherson, P., Belsky, D.W., Corcoran, D.L., Hammerle, M., Harrington, H., Hogan, S., Meier, M.H., Polanczyk, G.V., Poulton, R., Ramrakha, S., Sugden, K., Williams, B., Rohde, L.A., and Caspi, A. (2015). Is adult ADHD a childhood-onset neurodevelopmental disorder? Evidence from a 4-decade longitudinal cohort study. Am. J. Psychiatry 172: 967-977.

Moncrieff, J., and Timimi, S. (2010). Is ADHD a valid diagnosis in adults? No. BMJ 340: c547

Pelsser, L.M., Frankena, K., Toorman, J., and Rodrigues Pereira, R. (2017). Diet and ADHD, reviewing the evidence: a systematic review of metaanalyses of double-blind placebo-controlled trials evaluating the efficacy of diet interventions on the behavior of children with ADHD. PLoS One 12: e0169277.

Polanczyk, G., Silva de Lima, M., Lessa Horta, B., Biederman, J., and Rohde, L.A. (2007). The worldwide prevalence of ADHD: a systematic review and metaregression analysis. Am. J. Psychiatry 164: 942-948.

Poulton, A., and Nanan, R. (2017). Subcortical brain volume differences in participants with attention deficit hyperactivity disorder in children and adults. Lancet Psychiatry 4: 437-438.

Rijlaarsdam, J., Cecil, C.A., Walton, E., Mesirow, M.S., Relton, C.L., Gaunt, T.R., McArdle, W., and Barker, E.D. (2017). Prenatal unhealthy diet, insulin-like growth factor 2 gene (IGF2) methylation, and attention deficit hyperactivity disorder symptoms in youth with early-onset conduct problems. J. Child Psychol. Psychiatry 58: 19-27.

Rios-Hernandez, A., Alda, J.A., Farran-Codina, A., Ferreira-García, E., and Izquierdo-Pulido, M. (2017). The Mediterranean diet and ADHD in children and adolescents. Pediatrics 139: e20162027.

Sarris, J., Logan, A.C., Akbaraly, T.N., Amminger, G.P., Balanzá-Martínez, V., Freeman, M.P., Hibbeln, J., Matsuoka, Y., Mischoulon, D., Mizoue, T., Nanri, A., Nishi, D., Ramsey, D., Rucklidge, J.J., Sanchez-Villegas, A., Scholey, A., Su, K.-P., and Jacka, F.N. (2015). Nutritional medicine as mainstream in psychiatry. Lancet Psychiatry 2: 271-274.

Shaw, M., Hodgkins, P., Caci, H., Young, S., Kahle, J., Woods, A.G., and Arnold, L.E. (2012). A systematic review and analysis of long-term outcomes in attention deficit hyperactivity disorder: effects of treatment and non-treatment. BMC Medicine 10: 99.

Smith, M. (2017). Hyperactive around the world? The history of ADHD in global perspective. Soc. Hist. Med. 30: 767-787.

Sontag, T.A., Tucha, O., Walitza, S., and Lange, K.W. (2010). Animal models of attention deficit/hyperactivity disorder (ADHD): a critical review. Atten. Defic. Hyperact. Disord. 2: 1-20.

Storeb $\varnothing$, O.J., Faltinsen, E., Zwi, M., Simonsen, E., and Gluud, C. (2018). The jury is still out on the benefits and harms of methylphenidate for children and adolecents with attention deficit/hyperactivity disorder Clin. Pharmacol. Ther. 104: 606-609.

Swaab, D.F., and Boer, K. (2001). Functional teratogenic effects of chemicals on the developing brain. Fetal and neonatal neurology and neurosurgery, 3rd edition. In: Levene, M.I., Whittle, M.J., Chervenak, F.A., Punt, J., and Bennett, M.J. (Ed.). Elsevier, Churchill Livingstone, pp. 251-265.

Swanson, J.M., Arnold, L.E., Molina, B.S.G., Sibley, M.H., Hechtman, L.T., Hinshaw, S.P., Abikoff, H.B., Stehli, A., Owens, E.B., Mitchell, J.T., Nichols, Q., Howard, A., Greenhill, L.L., Hoza, B., Newcorn, J.H., Jensen, P.S., Vitiello, B., Wigal, T., Epstein, J.N., Tamm, L., Lakes, K.D., Waxmonsky, J., Lerner, M., Etcovitch, J., Murray, D.W., Muenke, M., Acosta, M.T., Arcos-Burgos, M., Pelham, W.E., and Kraemer, H.C. (2017) 
Young adult outcomes in the follow-up of the multimodal treatment study of attention-deficit/hyperactivity disorder: symptom persistence, source discrepancy, and height suppression. J. Child Psychol. Psychiatry 58: 663-678.

Thome, J., Ehlis, A.C., Fallgatter, A.J., Krauel, K., Lange, K.W., Riederer, P., Romanos, M., Taurines, R., Tucha, O., Uzbekov, M., and Gerlach, M. (2012). Biomarkers for attention-deficit/hyperactivity disorder (ADHD). A consensus report of the WFSBP task force on biological markers and the World Federation of ADHD. World J. Biol. Psychiatry 13: $379-400$.

Timimi, S., and Leo, J. (Ed.). (2009). Rethinking ADHD: from brain to culture. Palgrave Macmillan.

Valls-Pedret, C., Sala-Vila, A., Serra-Mir, M., Corella, D., de la Torre, R., Martínez-González, M.Á., Martínez-Lapiscina, E.H., Fitó, M., Pérez-Heras,
A., Salas-Salvadó, J., Estruch, R., and Ros, E. (2015). Mediterranean diet and age-related cognitive decline: a randomized clinical trial. JAMA Intern. Med. 175: 1094-1103.

Visser, J., and Jehan, Z. (2009). ADHD: a scientific fact or a factual opinion? A critique of the veracity of attention deficit hyperactivity disorder. Emotion. Behav. Difficult. 14: 127-140.

Wang, G.J., Volkow, N.D., Wigal, T., Kollins, S.H., Newcorn, J.H., Telang, F., Logan, J., Jayne, M., Wong, C.T., Han, H., Fowler, J.S., Zhu, W., and Swanson, J.M. (2013). Long-term stimulant treatment affects brain dopamine transporter level in patients with attention deficit hyperactive disorder. PLoS ONE 8: e63023.

Zhou, F., Wu, F., Zou, S., Chen, Y., Feng, C., and Fan, G. (2016). Dietary, nutrient patterns and blood essential elements in Chinese children with ADHD. Nutrients 8: E352. 